\title{
Strengthening Invariants by Symbolic Consistency Testing ${ }^{\star}$
}

\author{
Husam Abu-Haimed, Sergey Berezin, and David L. Dill \\ Stanford University \\ \{husam, berezin, dill\}@stanford.edu
}

\begin{abstract}
We describe a relatively simple method for strengthening invariants when verifying infinite-state hardware systems called symbolic consistency testing. The method requires a high-level symbolic simulator and a decision procedure for quantifier-free first-order logic. The user only needs to provide a small number of simple symbolic test vectors that expose internal inconsistencies in the system state. A verification system then uses symbolic simulation with these test vectors to strengthen the original invariant to an inductive one, which is discharged using $k$-step induction. The main advantage of our method is that the user input is usually very simple and intuitive, and the user does not need to be exposed to the actual complexity of the verification, which then proceeds completely automatically. The method is applied to several typical microarchitectures for cached memories.
\end{abstract}

\section{Introduction}

There are many potential advantages to verifying high-level descriptions of hardware systems, before commitments to implementation details such as data-path width are made. Errors that are best corrected by major design or architecture changes can be detected while there is still time to fix them properly, rather than patching a fundamentally-flawed design when it is too late to do anything else. Also, the verification effort is more valuable since results can be re-used for many different ways of implementing the design.

Unfortunately, most of the current practical formal verification methods, such as model checking, cannot be directly applied before implementation details are fixed, because they cannot deal with systems with unbounded numbers of states. The most widely used tools for formal verification of high-level system descriptions are interactive theorem provers, such as HOL or PVS [7]12], or even manual proof. However, even with steady improvement in interactive theorem provers, proofs can be very tedious.

\footnotetext{
* This research was supported by GSRC contract DABT63-96-C-0097-P00005, by National Science Foundation CCR-0121403, and by King Fahd University of Petroleum and Minerals, Saudi Arabia. The content of this paper does not necessarily reflect the position or the policy of GSRC, NSF, or the Government, and no official endorsement should be inferred.
} 
We describe a methodology, called symbolic consistency testing, that addresses one of the core problems in verifying non-trivial systems: computing inductive invariants. Finding invariants is a major problem even for most realistic finite-state systems; for relatively small finite-state systems the problem can be automatically solved by model checkers. Although there is no general-purpose algorithm to find inductive invariants automatically for infinite-state systems, it is possible to accumulate an inventory of incomplete, usually domain-specific techniques to help find them.

Symbolic consistency testing exploits symbolic simulation and a decision procedure for a quantifier-free fragment of first-order logic. The prototype implementation of the idea is based on the CVC tool [13], a general-purpose decision procedure for quantifier-free first-order logic, but the same ideas could be used with an interactive theorem prover if it has adequate support for efficient symbolic simulation and can effectively solve the logic formulas in question.

The user input for this verification method is the design to be verified, an invariant property to be proved (which is not inductive, in general), and a symbolic consistency test consisting of several short symbolic test vectors that will expose inconsistencies in the internal state of the system. The symbolic test vectors are symbolically simulated by the verification system to produce logical terms, which are then used to strengthen the original invariant to make it inductive. The resulting invariant may be very large and complex, but most of it is constructed automatically, so the user does not need to see it. This new invariant is then proven by a $k$-step induction (for some fixed $k$ that depends on the design).

We demonstrate the use of the technique to verify the microarchitectures of cached memory systems. No properties are assumed about the addresses and values of the memory system, so any proved properties will hold for a large number of possible implementations of the microarchitecture. For these systems, the symbolic consistency tests involve reading a small number of distinct symbolic addresses.

The verification problem is to compare a memory system microarchitecture with an idealized view of the memory as a simple uncached array. The microarchitecture and the array can be regarded as a single system consisting of the two subsystems running in parallel with identical inputs. Given executable descriptions of the memory system microarchitecture and the array, the property can be formalized as an invariant, which is usually not inductive, and requires some consistency condition on this combined system for every state that is reachable from some given initial state.

The resulting predicate describes the behavior of the system over several cycles of execution. Therefore, it is helpful to extend the basic method of proving invariants to span several cycles as well, so invariant resulting from consistency testing is proved using $k$-step induction, where $k$ is some constant that depends on the design.

Many techniques have been developed in the literature for finding invariants automatically 914162 111]. In spite of the sophistication of these techniques, 
the process of finding invariants is still mostly manual. Symbolic simulation has been used as a tool to reduce the manual effort in constructing the invariants 14 15:17.

Manna and Pnueli 9] showed methods based on bottom-up techniques for verifying temporal properties of reactive systems, which are then used to extract assertions implied by the transition relation. These assertions are used to strengthen the invariant.

$\mathrm{Su}$ et al. 14 presented some heuristics that automatically generate some of the invariants needed in processor verification based on syntactic manipulations of the transition function of the design. These techniques are classified as bottomup methods.

Among the most successful approaches to proving invariants and other properties for infinite-state systems are those based on predicate abstraction $[3,6,8,4,5]$. In predicate abstraction, the infinite-state system is abstracted into a finite system using a set of predicates on the system variables. These predicates are usually provided by the user. If the properties of interest hold for the finite abstract system, they hold for the infinite system.

Another class of techniques called top-down methods start with the property to be proved and use backward propagation with different heuristics to strengthen that property. Combinations of the top-downs and bottom-up techniques 162 have also been developed.

\section{Induction on Time}

We model a hardware design as a transition system

$$
T=\left(S, s_{0}, N, R, D_{\text {in }}, D_{\text {out }}\right),
$$

where $S$ is a non-empty (and possibly infinite) set of states, $s_{0} \in S$ is the initial state, $D_{\text {in }}$ and $D_{\text {out }}$ are the domains of inputs and outputs, $N: S \times D_{\text {in }} \rightarrow S$ is the transition function, and $R: S \times D_{\text {in }} \rightarrow D_{\text {out }}$ is the output function. Intuitively, a run of a transition system on an input sequence $\alpha^{\ell} \in D_{\text {in }}^{*}$ of length $\ell$ is a sequence of states $\pi=s_{0} s_{1} \ldots s_{l}$ starting from the initial state $s_{0}$, such that $s_{i+1}=N\left(s_{i}, \alpha_{i}\right)$ for all $0 \leq i \leq \ell-1$, producing a sequence of outputs $\xi^{\ell} \in D_{\text {out }}^{*}$, where each $\xi_{i}=R\left(s_{i}, \alpha_{i}\right)$. We write $N\left(s, \alpha^{\ell}\right)$ to denote the final state of running $T$ on the input sequence $\alpha^{\ell}$ starting from the state $s$ :

$$
N\left(s, \alpha^{\ell}\right)=\underbrace{N(N(\ldots N}_{\ell}\left(s, \alpha_{0}\right), \alpha_{1}), \ldots, \alpha_{\ell-1}) .
$$

In particular, $N\left(s, \alpha^{\ell}\right)=s$ when $\ell=0$ (the input sequence is empty). It is important to note that a single transition in $T$ can actually represent a complex transaction in the real hardware implementation requiring multiple cycles of execution. Therefore, the next state function $N$ may have a quite complex definition. 
A state $s$ is called reachable in a transition system $T$, if there is an input sequence $\alpha^{\ell}$ such that $s=N\left(s_{0}, \alpha^{\ell}\right)$, where $s_{0}$ is the initial state of $T$. In this paper, we only consider safety properties, or invariants over the set of reachable states. We say that a transition system $T$ satisfies a safety property $Q(s)$, if $Q(s)$ holds for every reachable state $s$ of $T$ :

$$
\forall s \text {. reachable }(s) \Rightarrow Q(s)
$$

This can be equivalently rewritten as follows:

$$
\forall \ell, \alpha^{\ell} \cdot Q\left(N\left(s_{0}, \alpha^{\ell}\right)\right)
$$

The conventional way of proving (2) is by induction on time, when $Q$ is first shown to hold in the initial state $s_{0}$, and then the transition function $N$ is shown to preserve $Q$ :

$$
\begin{aligned}
& Q\left(s_{0}\right) \\
& \forall s, \sigma . Q(s) \Rightarrow Q(N(s, \sigma)) .
\end{aligned}
$$

This simple induction, however, fails most of the time in practice, because the property we want to prove is not inductive and needs to be strengthened. Often, the problem of finding an inductive invariant is the hardest part of verification, which requires extensive manual guidance and depends greatly on the class of problems to be solved.

In the remainder of this section we introduce a new technique for strengthening an important class of invariants called functional equivalence and justify its soundness. The technique gives the user a simple recipe on how to collect the additional information about the design, and the rest of the verification proceeds completely automatically.

\subsection{Functional Equivalence}

We prove correctness of systems using the idea of functional equivalence. The problem is stated as follows. Given two systems, the concrete system $T^{c}$ (the system we want to verify) and the abstract system $T^{a}$ (which defines the required functionality of $T^{c}$ ), prove that $T^{c}$ is functionally equivalent to $T^{a}$. Two systems are said to be functionally equivalent if they produce the same sequence of outputs for the same sequence of inputs. Formally, this is expressed as follows.

The states $s^{c}$ and $s^{a}$ of $T^{c}$ and $T^{a}$ respectively are said to be functionally equivalent if both systems produce the same output for any given input in those states:

$$
\forall \lambda . R^{c}\left(s^{c}, \lambda\right)=R^{a}\left(s^{a}, \lambda\right) .
$$

The entire system $T^{c}$ is said to be functionally equivalent to $T^{a}$ if any reachable state $s^{c}$ of $T^{c}$ reached by some input sequence $\alpha^{\ell}$ is functionally equivalent to the corresponding state $s^{a}$ of $T^{a}$ reached by the same input sequence:

$$
\forall \ell, \alpha^{\ell}, \lambda . R^{c}\left(N^{c}\left(s_{0}^{c}, \alpha^{\ell}\right), \lambda\right)=R^{a}\left(N^{a}\left(s_{0}^{a}, \alpha^{\ell}\right), \lambda\right) .
$$




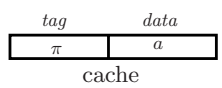

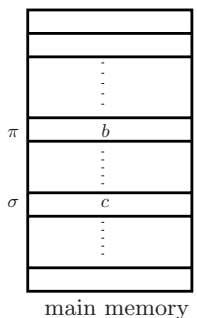

Concrete Module

(a)

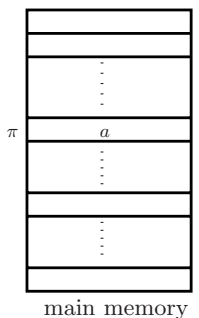

Abstract Module

(b)

Fig. 1. Memory Example

Effectively, we construct a product transition system

$$
T=\left(S^{c} \times S^{a},\left(s_{0}^{c}, s_{0}^{a}\right), N, R, D_{\text {in }}, D_{\text {out }}\right),
$$

where

$$
\begin{aligned}
& N\left(\left(s^{c}, s^{a}\right), \alpha\right)=\left(N^{c}\left(s^{c}, \alpha\right), N^{a}\left(s^{a}, \alpha\right)\right) \\
& R\left(\left(s^{c}, s^{a}\right), \alpha\right)=\left(R^{c}\left(s^{c}, \alpha\right), R^{a}\left(s^{a}, \alpha\right)\right)
\end{aligned}
$$

and define $Q(s)$ to be $\forall \lambda . R^{a}\left(s^{a}, \lambda\right)=R^{c}\left(s^{c}, \lambda\right)$. The functional equivalence property (5) then becomes $\forall \ell, \alpha^{\ell} . Q\left(N\left(\left(s_{0}^{c}, s_{0}^{a}\right), \alpha^{\ell}\right)\right)$, which is the same as formula (2). So, we can use the same induction principle given by (B) to prove the functional equivalence (5) of the two modules.

\subsection{Example: One-Line Cache}

Consider a small example of a read-only memory with a single-line cache given in figure 1(a). To verify the correctness of this design, we show that it is functionally equivalent to a simple (uncached) array of data in figure1(b). Since the memories are read-only, the input to both modules is the address, and the output is the data read from that address:

$$
\begin{aligned}
& D_{\text {in }}=\text { Addr } \\
& D_{\text {out }}=\text { Data }
\end{aligned}
$$

The transition systems $T^{c}$ and $T^{a}$ are defined as follows. The abstract state $s^{a}$ of $T^{a}$ is just an array $M$ indexed by Addr and holding the Data elements. The next state function $N^{a}$ is the identity function, and $R^{a}\left(s^{a}, \lambda\right)=M[\lambda]$. The concrete state $s^{c}$ of $T^{c}$ contains the state of the cache in addition to the same array $M$. Initially, in $s_{0}^{c}$, the cache is empty. The next state function $N^{c}\left(s^{c}, \lambda\right)$ adds the address $\lambda$ and the data stored under that address $M[\lambda]$ to the cache, 
yielding the new state. The output function $R^{c}\left(s^{c}, \lambda\right)$ is similar to $N^{c}$, except that it returns the data associated with the address $\lambda$.

Unfortunately, proving the functional equivalence of the two memories by simple induction fails. Consider a state in which the cache in the concrete memory contains the data $a$ for an address $\pi$, but the main memory has a different value $b$ under the same address. The two memories $T^{c}$ and $T^{a}$ are functionally equivalent in the current state $s$ if the abstract memory happens to contain $a$ for the address $\pi$, thus, the induction hypothesis $Q(s)$ is satisfied. However, transitioning to the next state by reading some address $\sigma \neq \pi$ brings $T^{c}$ to a new state $s^{\prime}$ where the address $\pi$ is no longer cached. Therefore, reading $\pi$ again yields $b \neq a$ (since it has to come from the main memory), which no longer agrees with $T^{a}$.

The induction fails in this case because it starts out from an inconsistent state, which is not reachable. The natural way to strengthen the invariant is to require the state to be consistent. In this example it means that the cached value must be the same as in the main memory (this property is called coherence).

Fortunately, there is a very simple way to test for consistency. The idea is to read from the cached address $\pi$, then from some other address, to make sure that the original value in the cache is replaced, then read from $\pi$ again, and require that the results of the first and the last reads are the same. The second read from $\pi$ forces the cache to reload the value from the main memory, hence, this effectively requires the originally cached value to be consistent with the main memory.

This consistency test is very intuitive, and can be easily constructed by the designer. In our approach, supplying this information is sufficient to strengthen the invariant and finish the verification completely automatically. So, the user can use the tool as a black box, without having to know the details of the algorithm, or be an expert in formal verification.

Notice that the consistency test spans several consecutive states of execution, which requires a more general $k$-step induction, which we define formally in the next section.

\section{$2.3 \quad k$-Step Induction}

Formally, in order to show $\forall \ell, \alpha^{\ell} . Q\left(N\left(s_{0}, \alpha^{\ell}\right)\right)$, it is sufficient to prove two formulas representing the base case and the induction step:

$$
\begin{aligned}
& \forall \ell<k \forall \alpha^{\ell} . Q\left(N\left(s_{0}, \alpha^{\ell}\right)\right) \\
& \forall s, \sigma^{k} .\left[\bigwedge_{\ell=0}^{k-1} \forall \alpha^{\ell} . Q\left(N\left(s, \alpha^{\ell}\right)\right)\right] \Rightarrow Q\left(N\left(s, \sigma^{k}\right)\right)
\end{aligned}
$$

In practice, it is possible to prove a stronger and a slightly simpler formula for the induction step (7):

$$
\forall s, \sigma^{k} \cdot \exists \alpha^{k-1} \cdot\left[\bigwedge_{\ell=0}^{k-1} Q\left(N\left(s, \alpha^{\ell}\right)\right)\right] \Rightarrow Q\left(N\left(s, \sigma^{k}\right)\right),
$$


where $\alpha^{\ell}$ is a prefix of $\alpha^{k-1}$ for all $\ell<k$. So, if there is a $k$ such that (6) and (8) hold, we conclude that (2) is true.

Note that formula (8) contains an existential quantifier, and since solving validity of existential first-order formulas is undecidable, some manual guidance may be necessary to eliminate the existential quantifiers. In section [3.1, we propose a new instantiation method which significantly reduces the required manual effort. Decidability is not an issue for the base case of the induction (6), since the universal fragment of the logic is decidable and is directly supported by CVC, our validity checker.

\subsection{Symbolic Simulation}

Intuitively, a symbolic simulation of a transition system $T$ is just like an ordinary simulation, only the input sequence $\alpha^{\ell}$ and the starting state $s$ are symbolic variables rather than constant values. Therefore, the resulting run over $\alpha^{\ell}$ becomes the sequence of symbolic terms representing the states of the run:

$$
s, N\left(s, \alpha^{1}\right), N\left(s, \alpha^{2}\right), \ldots, N\left(s, \alpha^{\ell}\right),
$$

where each $\alpha^{i}$ is a prefix of $\alpha^{\ell}$. One can think of such a symbolic run as "the most general run" of $T$ of length $\ell$, since any other run of the same length can be obtained from (9) by instantiating $\alpha^{\ell}$ with concrete values.

For practical examples, the next state and output functions $N(s, \alpha)$ and $R(s, \alpha)$ are explicitly defined as terms over $s$ and $\alpha$, and each symbolic state becomes a complex term. Moreover, real hardware circuits often require multiple cycles to complete a single operation, which in $T$ is treated as "one step" of execution. So, in reality, $N(s, \alpha)$ may denote the final state of a long sequence of intermediate, unobservable states of the real implementation. This fact significantly contributes to the size of the resulting state terms. The task of a symbolic simulator is to build the state terms efficiently, which in practice may be as large as hundreds of thousands of nodes.

The importance of symbolic simulation to formal verification is in the following observation. If a safety property $Q$ is proven for a symbolic run of length $\ell$, then $Q$ holds for any concrete run of length $\ell$ of $T$. This provides a method for proving formulas (6) and (8). Obviously, proving $Q(s)$ where $s$ is a huge term is a challenging task, and a powerful theorem prover or a validity checker engine is required. In this work, CVC is used both as a symbolic simulator and a validity checker.

\section{Soundness of Consistency Testing}

In section 2.2 we observed that $k$-step induction captures the consistency assumption that is necessary for the induction step to go through. Specifically, for the example in that section, the inductive step in the 2-step induction can be proven valid. From our experience, for virtually any memory design there exists 
some $k$ such that the correctness property for the design can be proven by $k$ step induction. The only remaining problem in the induction step (8) is that it contains existential quantifiers:

$$
\forall s, \sigma^{k} \exists \alpha^{k-1} \cdot\left[\bigwedge_{\ell=0}^{k-1} Q\left(N\left(s, \alpha^{\ell}\right)\right)\right] \Rightarrow Q\left(N\left(s, \sigma^{k}\right)\right) .
$$

The existential quantifiers need to be eliminated before the formula can be handed to the validity checker. In general, finding an instantiation for existential quantifiers is undecidable, and some manual guidance may be required. In our approach, we try to minimize the amount of user guidance and reduce it to expressing just the essence of the consistency property needed for the proof. For instance, in section 2.2 the idea behind the consistency test was to read from two distinct addresses to flush the cache and bring the system to a consistent state.

It is often the case that the important part of an instantiation for an existential formula is not a concrete term, but a certain property that the instantiation has to satisfy. Therefore, it is much easier and more intuitive to supply the property of importance explicitly and let the tool fill in the details. This idea can be formalized as a technique called predicate instantiation.

\subsection{Predicate Instantiation of Existential Quantifiers}

The existential quantifier in a formula of the form:

$$
\forall x . \exists y . \Psi(x, y)
$$

is usually eliminated by constructing a function $f(x)$ and replacing $y$ for $f(x)$ :

$$
\forall x . \Psi(x, f(x)) .
$$

However, constructing such a function explicitly for memory examples is a very tedious process. Instead, we apply a different technique which we call predicate instantiation. The idea is to find a predicate or a formula $\Phi(x, y)$ such that the following two formulas can be proven valid:

$$
\begin{aligned}
& \forall x . \exists y . \Phi(x, y) \\
& \forall x . \forall y . \Phi(x, y) \Rightarrow \Psi(x, y) .
\end{aligned}
$$

It is not hard to derive that the validity of (11) and (12) imply the validity of (10).

Let $P(s, \lambda)$ stand for $R^{c}\left(s^{c}, \lambda\right)=R^{a}\left(s^{a}, \lambda\right)$. When applying $k$-step induction to the example in section 2.2 (where $k=2$ ), the inductive step (8) becomes

$$
\forall s . \forall \sigma^{2} . \exists \alpha .\left[\forall \beta_{0} . P\left(s, \beta_{0}\right) \wedge \forall \beta_{1} . P\left(N(s, \alpha), \beta_{1}\right)\right] \Rightarrow \forall \lambda . P\left(N\left(s, \sigma^{2}\right), \lambda\right) .
$$

Pulling all the quantifiers to the top level yields the following:

$$
\forall s . \forall \sigma^{2} . \forall \lambda . \exists \alpha . \exists \beta^{2} .\left[P\left(s, \beta_{0}\right) \wedge P\left(N(s, \alpha), \beta_{1}\right)\right] \Rightarrow P\left(N\left(s, \sigma^{2}\right), \lambda\right) .
$$


Recall that in order to test for consistency, we must read from the address $\lambda$ to test if $P(s, \lambda)$ is true, then read from some other address $\alpha$ to get $N(s, \alpha)$, and then read from $\lambda$ again to test if $P(N(s, \alpha), \lambda))$ is true. If $P(s, \lambda)$ and $P(N(s, \alpha), \lambda)$ are true, then the memory system is consistent for this example. These requirements can be expressed by the following formula:

$$
\Phi: \quad \beta_{0}=\beta_{1}=\lambda \wedge \alpha \neq \lambda,
$$

which we use for predicate instantiation of existential quantifiers in (13):

$$
\begin{aligned}
& \forall s \forall \sigma^{2} \forall \lambda . \exists \alpha \exists \beta^{2} . \Phi \\
& \forall s \forall \sigma^{2} \forall \lambda . \forall \alpha \forall \beta^{2} .\left[\Phi \wedge P\left(s, \beta_{0}\right) \wedge P\left(N(s, \alpha), \beta_{1}\right)\right] \Rightarrow P\left(N\left(s, \sigma^{2}\right), \lambda\right) .
\end{aligned}
$$

The formula (15) is trivially true if the address space $D_{\text {in }}$ has more than one address, and (16) is shown to be valid by CVC, our automatic validity checker.

Note that to use the instantiation predicate, one still needs to prove an existential formula (11), which is undecidable in general. However, for all of the memory examples that we have tried, the resulting formula was simple enough to be solved automatically by a theorem prover. The theorem prover we used was Otter [10] which took a fraction of a second to prove each of these formulas.

\section{Examples}

To illustrate the use of the methodology described above on practical examples, we applied it to several memory designs with different cache architectures. The write-through direct mapped cache with a parameterized cache size is discussed in detail in section 4.1, and its extension to the multi-level cache design is given in section 4.2.

As we mentioned earlier, the base case of the inductive proof falls into a decidable fragment of the first-order logic, and does not present a problem in verification. Therefore, in the following examples, we concentrate only on proving the induction step.

\subsection{Write-through Direct Mapped Cache}

The memory design given in figure 2(a) is a generalization of the simple example in section 2.2 to a read/write memory with a write-through direct mapped cache and an arbitrary number of cache lines. The figure describes only the high level organization of the system. There are several intermediate registers and buffers in the system that we do not show to simplify the discussion. The system also has a state machine that consists of 6 states controlling the cache in addition to a 3 -state state machine that controls the main memory. Simulating a read or a write transaction in this system can take up to 10 cycles. An invariant for such a system needs to describe the values of the cache, the main memory, the intermediate registers, and the state machines at the different cycles of the transaction. That can be a very complex invariant. In this section we show how 

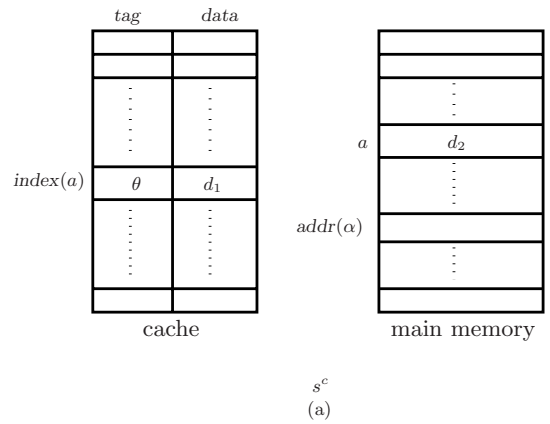

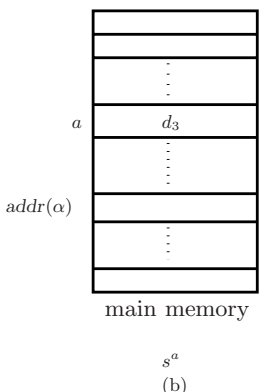

(b)

Fig. 2. Direct Mapped Cache

a simple symbolic consistency test for this system helps us avoid this complexity. The consistency test we show was enough for the induction step of the proof to go through.

The inputs to the memory consist of the read/write flag, the address, and the data in the case of the write flag. On the read input, the memory returns the requested data as an output, and write produces no output, which we denote by a special symbol $\perp$. Formally, we define the input and output domains of the transition system $T^{c}$ representing the design as follows:

$$
\begin{aligned}
& D_{\text {in }}=(\{\text { read }\} \times \text { Addr }) \cup(\{\text { write }\} \times \text { Addr } \times \text { Data }) \\
& D_{\text {out }}=\text { Data } \cup\{\perp\}
\end{aligned}
$$

As in section 2.1, we define the correctness of $T^{c}$ to be the functional equivalence between $T^{c}$ and the reference model $T^{a}$ (figure 2(b)), which is simply an array of data. We refer to $T$ as the product of $T^{c}$ and $T^{a}$; so a state $s$ of $T$ is a pair of states $\left(s^{c}, s^{a}\right)$ of the two systems.

For an input $\lambda=\langle\mathrm{rw}, a[, d]\rangle$, we write $r w(\lambda)$ and $\operatorname{add} r(\lambda)$ to denote the $\mathrm{read} /$ write flag and the address parts of $\lambda$ respectively. Each address $a$ consists of two components: $\operatorname{index}(a)$ and $\operatorname{tag}(a)$. The index part is used to identify the cache line (so, the cache array is indexed by index $(a)$ ), and the tag is stored in the cache along with the data to reconstruct the complete address.

Following our methodology, a consistency test needs to be constructed for $T^{c}$, which in this case means that the data stored in every cache line is coherent with the main memory. Similarly to the example in section 2.2 , consistency of each cache line can be checked by first reading the cached address $a=\operatorname{addr}(\lambda)$, then some other address $a^{\prime}=\operatorname{addr}(\alpha)$ with the same index as $a$ to replace the data in that cache line, then read from $a$ again and compare the result with the first read. This intuitive description is translated into the following formula:

$$
\begin{aligned}
\Phi \equiv & \beta_{0}=\beta_{1}=\lambda \\
& \wedge \operatorname{index}(\operatorname{addr}(\alpha))=\operatorname{index}(\operatorname{addr}(\lambda)) \wedge \operatorname{tag}(\operatorname{addr}(\alpha)) \neq \operatorname{tag}(\operatorname{addr}(\lambda)) .
\end{aligned}
$$

This is all the user needs to supply to the tool, and the rest is done completely automatically. 
Since three reads are required by the consistency test, two of which are consecutive (from $\alpha$ and $\beta_{1}$ ), 2-step induction is used. The formula (13), which is the form of the induction step we need to prove, becomes

$$
\forall s, \sigma^{2}, \lambda, \exists \alpha, \beta^{2} .\left[P\left(s, \beta_{0}\right) \wedge P\left(N(s, \alpha), \beta_{1}\right)\right] \Rightarrow P\left(N\left(s, \sigma^{2}\right), \lambda\right),
$$

where $P(s, \lambda) \equiv R^{c}\left(s^{c}, \lambda\right)=R^{a}\left(s^{a}, \lambda\right)$.

Observe, that when $\lambda=\langle$ write, $a, d\rangle$, formula (17) is trivially true, since $R^{c}\left(N^{a}\left(s^{a}, \sigma^{2}\right), \lambda\right)=R^{a}\left(N^{a}\left(s^{a}, \sigma^{2}\right), \lambda\right)=\perp$. Therefore, we only need to consider the case when $\lambda=\langle$ read, $a\rangle$.

Instantiating (17) with the predicate $\Phi$ above yields the following:

$$
\begin{aligned}
& \forall s, \sigma^{2}, \lambda, \exists \alpha, \beta^{2} . \Phi \\
& \forall s, \sigma^{2}, \lambda, \forall \alpha, \beta^{2} .\left[\Phi \wedge P\left(s, \beta_{0}\right) \wedge P\left(N(s, \alpha), \beta_{1}\right)\right] \Rightarrow P\left(N\left(s, \sigma^{2}\right), \lambda\right) .
\end{aligned}
$$

Formula (18) is valid whenever there exists more than one tag for the same index (which is the case for any real design), and (19) is proven valid by our validity checker CVC.

Recall, that the next state function in this example requires 10 cycles of symbolic simulation to compute. Therefore, the formula for the 2-step induction involves 20 cycles of symbolic simulation total. Moreover, due to the intermediate buffers, simply requiring that the cached value is consistent with the main memory is not enough for the invariant to become inductive.

\subsection{Two-Level Write-through Direct-Mapped Cache}

The system we deal with here has two levels of cache, each one of these two levels has an organization similar to that in the previous section. A transaction in this system can take up to 17 cycles. For this example, two levels of cache flushing are required for the consistency test, and therefore, we need 3-step induction. First, we find an address $\alpha_{0} \neq \lambda$ which maps to the same line of the L1 cache: $\operatorname{index}_{1}\left(\alpha_{0}\right)=\operatorname{index}_{1}(\lambda)$, and read from $\lambda$, then $\alpha_{0}$, then $\lambda$ again. This ensures that if $\lambda$ is cached both in L1 and L2, the cached data is the same in both caches. Next, we find another address $\alpha_{1} \neq \lambda$ such that $\alpha_{1}$ and $\lambda$ map to the same cache lines both in L1 and L2:

$$
\operatorname{index}_{1}\left(\alpha_{1}\right)=\operatorname{index}_{1}(\lambda) \wedge \operatorname{index}_{2}\left(\alpha_{1}\right)=\operatorname{index}_{2}(\lambda) .
$$

Reading from $\lambda$, then $\alpha_{1}$, then $\lambda$ again clears both caches at the same time and forces the data under the address $\lambda$ to be fetched from the main memory. Putting it all together yields the following instantiation predicate:

$$
\begin{gathered}
\Phi \equiv \quad \beta_{0}=\beta_{1}=\beta_{2}=\lambda \wedge \lambda \neq \alpha_{0} \neq \alpha_{1} \neq \lambda \wedge \operatorname{index}_{1}\left(\alpha_{0}\right)=\operatorname{index}_{1}(\lambda) \\
\wedge \operatorname{index}_{1}\left(\alpha_{1}\right)=\operatorname{index}_{1}(\lambda) \wedge \operatorname{index}_{2}\left(\alpha_{1}\right)=\operatorname{index}_{2}(\lambda) .
\end{gathered}
$$

Notice, that $\Phi$ is an acceptable predicate (i.e. it satisfies (11)) only under the assumption that any cache line has several distinct addresses that map to it 
(three for L1 and two for L2), and, moreover, that for any address $\lambda$ there is another address $\alpha_{1}$ which maps to exactly the same cache lines as $\lambda$ throughout all the cache levels. Both assumptions are quite reasonable for real designs.

As before, the next state function $N$ for this example requires 17 cycles of symbolic execution, yielding 51 cycles total for the 3-step induction. Again, the actual inductive invariant requires much more information about the intermediate buffers and registers than the high-level description above provides.

This consistency test can be generalized to memory designs with $n$-level caches for any concrete $n$, and verified using $(n+1)$-step induction.

We applied our approach to many other examples with different cache organizations such as two-way set-associative caches, and write-back caches. In all cases, the instantiation predicate was very compact and easy to construct, and no other manual guidance was required to complete the verification.

One exception is the two-way set-associative cache, where an additional modest manual intervention reduced the original 5-step induction to 3 steps in order to speed up the validity checking. The additional property is that the same address can be cached in at most one cache line.

\section{Conclusion}

This paper addresses one of the most difficult problems in verifying safety properties for infinite-state systems: finding inductive invariants. This problem is undecidable in general and often requires substantial ingenuity and manual guidance from the user.

The proposed methodology reduces the user's burden to understanding the bare essence of the inductive invariant and constructing a relatively simple predicate to help the otherwise automatic tool to finish the proof of the invariant.

Although much simpler than finding an inductive invariant manually, constructing the instantiation predicate can still be a challenging task. However, for cached memories, which is an important class of hardware designs, there is a practical strategy which greatly helps the user to construct such a predicate. As we have observed, the main reason why the invariant is inductive is because any reachable state is consistent with itself; that is, the cached data must always be consistent with the main memory. Such a consistency test is often intuitive and easy to construct for a person familiar with the design, and is sufficient to complete the verification automatically. This methodology is actually not restricted to only cached memories, and is applicable to many other classes of designs.

The approach is still in its development stage, and a lot remains to be done. One obvious problem is that the formulas resulting from symbolic simulation are often enormous, which requires a powerful validity checker. Much research needs to be done to optimize the validity checker to the class of formulas that arise in this approach.

Another direction of research is to find heuristics to help the user find the instantiation predicates automatically, and to expand the class of designs this approach can handle. 
Acknowledgements. The authors would like to thank Aaron Stump, Clark Barrett and Satyaki Das for their help and support with the verification tools, William McCune for his help with Otter, and the anonymous reviewers for the insightful comments on the paper.

\section{References}

1. Saddek Bensalem, Yassine Lakhnech, and Hassen Saidi. Powerful techniques for the automatic generation of invariants. In Computer Aided Verification, 1996.

2. Nikolaj Bjørner, Anca Browne, and Zohar Manna. Automatic generation of invariants and intermediate assertions. In Theoretical Computer Science, 1997.

3. Michael Colon and Tomas E. Uribe. Generating finite-state abstractions of reactive systems using decision procedures. In Computer Aided Verification, volume 1427 of LNCS, pages 293-304. Springer Verlag, 1998.

4. Satyaki Das and David L. Dill. Successive approximation of abstract transition relations. In Proceedings of the Sixteenth Annual IEEE Symposium on Logic in Computer Science, 2001. June 2001, Boston, USA.

5. Satyaki Das and David L. Dill. Counter-example based predicate discovery in predicate abstraction. In Formal Methods in Computer-Aided Design. SpringerVerlag, November 2002.

6. S. Graf and H. Saidi. Construction of abstract state graphs with PVS. In Orna Grumberg, editor, Computer-Aided Verification, CAV '97, volume 1254 of Lecture Notes in Computer Science, pages 72-83, Haifa, Israel, June 1997. Springer-Verlag.

7. The HOL System Tutorial, 1994. http://www.cl.cam.ac.uk/Research/HVG/HOL/.

8. D. Lesens and H. Saïdi. Automatic verification of parameterized networks of processes by abstraction. In the 2nd International Workshop on the Verification of Infinite State Systems (INFINITY'97), July 1997.

9. Zohar Manna and Amir Pnueli. Temporal Verification of Reactive Systems: Safety. Springer-Verlag, 1993.

10. William W. McCune. Otter 3.0 reference manual and guide. Technical report, ANL, 1994.

11. John Rushby. Integrated formal verification: Using model checking with automated abstraction, invariant generation, and theorem proving. In 5th SPIN workshop, 1999.

12. N. Shankar, S. Owre, and J. M. Rushby. PVS Tutorial. Computer Science Laboratory, SRI International, Menlo Park, CA, February 1993. Also appears in Tutorial Notes, Formal Methods Europe '93: Industrial-Strength Formal Methods, pages 357-406, Odense, Denmark, April 1993.

13. A. Stump, C. Barrett, and D. Dill. CVC: a Cooperating Validity Checker. In 14th International Conference on Computer-Aided Verification, 2002.

14. Jeffrey X. Su, David L. Dill, and Clark W. Barrett. Automatic generation of invariants in processor verification. In FMCAD, 1996.

15. Jeffrey X. Su, David L. Dill, and Jens U. Skakkebæk. Formally verifying data and control with weak reachability invariants. In FMCAD, 1998.

16. A. Tiwari, H. Rueß, H. Saidi, and N. Shankar. A technique for invariant generation. In Tools and Algorithms for the Construction and Analysis of Systems, 2001.

17. Miroslav N. Velev and Randal E. Bryant. Formal verification of superscalar microprocessors with multicycle functional units, exceptions, and branch prediction. In Design Automation Conference (DAC), 2000. 